\title{
Analytic Continuation of Group Representations
}

\author{
R. HermanN * \\ Argonne National Laboratory, Argonne, Illinois \\ Received January 26, 1966
}

\begin{abstract}
Some general methods for analytically continuing group representations are presented. In favorable cases, this enables one to recognize the generalization of the "principal", "discrete" and "supplementary" series.
\end{abstract}

\section{Introduction}

In [4] we pointed out there is a natural way of "analytically continuing" certain types of unitary representations of non-compact semisimple Lie groups to finite dimensional representations, i.e. to unitary representations of the compact real form of the group. This phenomenon is quite well-known in case the group is $S L(2, R)$, and is associated in that case with the "Regge pole" ideas of elementary particle physics. Y. NE'EMAN has suggested that the extension of this idea to the other Lie groups used to classify elementary particles (e.g. $S U(3))$ might have physical applications. With these possibilities in the background, this paper is devoted to the mathematical problems. In particular, we will extend the method used in [4] for analytic continuation (which required that the representation satisfied the "Gell-Mann formula") to considerably more general and useful situations. On the other hand, in order to make the methods understandable to a non-specialist, we have not tried to push them to their natural limits.

Let $G$ be a Lie algebra, and let $H$ be a Hilbert space. Denote the abstract typical elements of $G$ by $X, Y$, etc. Suppose that $X \rightarrow X^{\prime}$ defines a representation of $\mathrm{G}$ by skew-Hermitian operators on $H$. An analytic continuation of this representation is a family $G^{\lambda}$ of representations $X \rightarrow X^{\lambda}$ of $\mathbf{G}$ by operators depending complex-analytically on the parameter $\lambda$, reducing to the given one for $\lambda=0$. In this paper, we will consider such continuations defined by "infinitesimal multipliers". These, will be shown to be skew-Hermitian for pure imaginary values of $\lambda$, which will define the analog of the "principal series" known for $S L(2, R)$ and other classical groups, [1,2]. In [4] we showed that a special type of such analytic continuations is reducible for a discrete set of real values of $\lambda$. Here, we shall analyze the conditions for such behavior more

* Work performed under the auspices of the United States Atomic Energy Commission. 
precisely in terms of the creation-annihilation operator formalism described in [5]. Further, we shall analyze the conditions that the operators $X$ can be made skew-Hermitian for certain other real values of $\lambda$ by a modification of the inner product in $H$, leading to a generalization of the "supplementary" and "discrete" series, as they are known for $S L(2, R)$.

\section{Analytic continuation by means of multipliers}

Let $G$ be a Lie algebra and suppose that $X \rightarrow X^{\prime}$ defines a representation of $G$ by skew-Hermitian operators on a Hilbert space $H$. Suppose that to each $X \in \mathbb{G}$ we assign another operator $X^{\prime \prime}$ on $H$, and form the operator $X^{\prime}+X^{\prime \prime}$. Let us find the conditions that the assignment $X \rightarrow X^{\prime}+X^{\prime \prime}$ also defines a representation of G. First, of course, we must have:

$$
(X+Y)^{\prime \prime}=X^{\prime \prime}+Y^{\prime \prime} \quad \text { for } \quad X, Y \in \mathbf{G} .
$$

Also, $\left[X^{\prime}+X^{\prime \prime}, Y^{\prime}+Y^{\prime \prime}\right]$ should be $[X, Y]+[X, Y]^{\prime \prime}$ i.e.

$$
[X, Y]^{\prime \prime}=\left[X^{\prime \prime}, Y^{\prime}\right]+\left[X^{\prime}, Y^{\prime \prime}\right]+\left[X^{\prime \prime}, Y^{\prime \prime}\right] \text { for } X, Y \in \mathbf{G} \text {. }
$$

Such an assignment of operators to elements of $G$ is called a system of (infinitesimal) multipliers for the original representation of $G$. If we regard (2.2) as a system of equations for the operators $X^{\prime \prime}$, note that they are not necessarily linear. In fact, the condition that they be linear is:

$$
\left[X^{\prime \prime}, Y^{\prime \prime}\right]=0 \text { for } X, Y \in \mathbf{G} .
$$

Then, (2.2) takes the form

$$
[X, Y]^{\prime \prime}=\left[X^{\prime \prime}, Y^{\prime}\right]+\left[X^{\prime}, Y^{\prime \prime}\right] .
$$

We shall mainly work with the multiplier systems satisfying (2.3), since they seem to be the simplest and most interesting case.

Notice that if $X \rightarrow X^{\prime \prime}$ satisfies $(2.3-4)$, so does $X \rightarrow \lambda X^{\prime \prime}$, where $\lambda$ is any complex number. Thus, we can form the operators

$$
X^{\lambda}=X^{\prime}+\lambda X^{\prime \prime}
$$

and obtain representations of $\mathbf{G}$ depending complex-analytically on the parameter $\lambda$. If $X^{\prime \prime}$ is Hermitian, note that $X^{\lambda}$ is skew-Hermitian if and only if $\lambda$ is pure imaginary, so that we obtain in this way the "principal series".

As a side point, let us inquire about the limit as $\lambda \rightarrow \infty$ (in the sense explained in [3]) of the Lie algebra $\mathbf{G}^{\lambda}$ of operators spanned by the $X^{\lambda}$. Let $\mathbf{K}$ be the set of $X \in \mathbf{G}$ such that $X^{\prime \prime}=0$. Notice that $\mathbf{K}$ is a subalgebra of $\mathbf{G}$. Suppose we split up $\mathbf{G}$ as $\mathbf{K} \oplus \mathbf{P}$. Then, for $X \in \mathbf{P}$, $\lim X^{\lambda} / \lambda=X^{\prime \prime}$. Hence:

The limit as $\lambda \rightarrow \infty$ of the algebras $G^{\lambda}$ is the algebra of operators generated by $X^{\prime}$, for $X \in \mathbf{K}$, and the $X^{\prime \prime}$, for $X \in \mathbf{P}$. 


\section{Changing the inner product to make representations unitary}

The process described by the title of this section is a common way of constructing unitary representations. For example, suppose that we start off with the Hilbert space of square integrable functions $f(x)$ of a real variable $x$, with the inner product given by

$$
\langle f \mid g\rangle=\int f(x)^{*} g(x) d x .
$$

Suppose that $A$ is an operator given by a kernel $K\left(x, x^{\prime}\right)$, i.e.

$$
A f(x)=\int K\left(x, x^{\prime}\right) f\left(x^{\prime}\right) d x^{\prime} .
$$

Define a new inner product by the formula:

Explicitly,

$$
\{f \mid g\}=\langle f \mid A g\rangle .
$$

$$
\{f / g\}=\iint K\left(x, x^{\prime}\right) f(x)^{*} g\left(x^{\prime}\right) d x d x^{\prime} .
$$

We recognize that this is the type of inner product used to construct the "supplementary series" of unitary representations of $S L(2, R)[1]$. It is also used in quantum field theory: For example, the solutions of a Lorentz invariant differential equation (such as the Dirac equation) should, for the purposes of second quantization, form a Hilbert space in which the Poincaré group acts via unitary transformations. The usual integration-type inner product is unsatisfactory because the Lorentz group is non-compact and does not leave invariant a positive definite inner product on the spinors. It must be modified by changing it with an operator $A$ as explained above.

Let us work out the general formalities. Suppose that $H$ is a Hilbert space, with inner product $\left\langle\psi / \psi^{\prime}\right\rangle$. If $A$ is an operator on $H$, let $A^{*}$ be its adjoint, i.e.

$$
\left\langle\psi \mid A \psi^{\prime}\right\rangle=\left\langle A^{*} \psi \mid \psi^{\prime}\right\rangle .
$$

Suppose that we define a new inner product by the rule:

$$
\left\{\psi / \psi^{\prime}\right\}=\left\langle\psi / A \psi^{\prime}\right\rangle \text {. }
$$

The condition that this also be an Hermitian inner product is:

i.e.

$$
\left\{\psi / \psi^{\prime}\right\}=\left\{\psi^{\prime} \mid \psi\right\}^{*},
$$

$$
\left\langle\psi \mid A \psi^{\prime}\right\rangle=\left\langle\psi^{\prime} \mid A \psi\right\rangle^{*}=\left\langle A^{*} \psi^{\prime} \mid \psi\right\rangle=\left\langle\psi \mid A^{*} \psi^{\prime}\right\rangle,
$$

or

$$
A \text { is Hermitian, i.e. } A=A^{*} \text {. }
$$

Suppose now that $\mathrm{G}$ is a Lie algebra of operators on $H$. Let us look for the condition that $X \in \mathbb{G}$ be skew-Hermitian with respect to the $\{\psi / \psi\}$-inner product:

$$
0=\left\{X \psi / \psi^{\prime}\right\}+\left\{\psi \mid X \psi^{\prime}\right\}=\left\langle X \psi / A \psi^{\prime}\right\rangle+\left\langle\psi^{\prime} A X \psi^{\prime}\right\rangle
$$


or

$$
X^{*} A+A X=0 \text { for } X \in \mathbf{G} .
$$

Suppose, for example, that $A^{-1}$ exists. Then, (3.2), takes the form

$$
X=-A^{-1} X^{*} A
$$

Notice that $X \rightarrow-X^{*}$ is also a representation of $\mathbf{G}$. Hence, the existence of a new Hermitian inner product with respect to which $G$ is an algebra of skew-Hermitian operators is associated with an equivalence between this representation and its Hermitian adjoint.

\section{Reducibility for analytically continued representations}

Suppose that $G$ is a Lie algebra, $H$ a Hilbert space, and that for each complex number $\lambda$ the operator assignment

$$
X \rightarrow X^{\prime}+\lambda X^{\prime \prime}
$$

forms a representation of $G$, with $X^{\prime}$ skew-Hermitian, and $X^{\prime \prime}$ Hermitian. We want to describe conditions that the representation be reducible for certain real values of $\lambda$, following the pattern described in [4], using the creation-annihilation operator formalism described in [5].

Suppose that $H$ is the direct sum $H^{0}+H^{1}+H^{-1}+H^{2}+H^{-2}+\ldots$ of mutually orthogonal subspaces, and that each of the operators $X^{\prime}, X^{\prime \prime}$ applied to $\psi \in H^{r}, r=0, \pm 1, \ldots$, connects at most to $H^{r+1}, H^{r}$ and $H^{r-1}$. Thus, $X^{\prime}$ and $X^{\prime \prime}$ can be written in the following form:

$$
\begin{aligned}
X^{\prime} & =X^{\prime+}-X^{\prime}-+X^{\prime 0} \\
X^{\prime \prime} & =X^{\prime \prime}++X^{\prime \prime}-+X^{\prime \prime 0},
\end{aligned}
$$

where $X^{\prime+}$ and $X^{\prime \prime}+$ map $H^{r}$ into $H^{r+1}, X^{\prime-}$ and $X^{\prime \prime}$ - are their adjoints, and $X^{\prime 0}, X^{\prime \prime} 0$ map $H^{r}$ into $H^{r}$. This way of decomposing $X^{\prime}$ and $X^{\prime \prime}$ makes the following result obvious.

Theorem 4.1. $\mathrm{G}^{\lambda}$ leaves invariant the subspace

if and only if:

$$
H^{a, b} \equiv H^{a}+H^{a+1}+\cdots+H^{b}(a \leqq b)
$$

$$
\begin{aligned}
& \left(X^{\prime+}+\lambda X^{\prime \prime+}\right) H^{a}=0 \\
& \left(X^{\prime-}+\lambda X^{\prime \prime}-\right) H^{b}=0, \text { for all } X \in \mathbf{G} .
\end{aligned}
$$

Now suppose that $\lambda_{0}, \lambda_{1}, \lambda_{-1}, \lambda_{2}, \lambda_{-2}, \ldots$ is a sequence of real numbers such that:

Then also:

$$
\left(X^{\prime+}+\lambda_{r} X^{\prime \prime}\right)\left(H^{r}\right)=0 \text { for all } r, \text { all } X \in \mathbf{G} .
$$

$$
\left(X^{\prime}-+\lambda_{r} H^{\prime \prime}-\right)\left(H^{r+1}\right)=0 \text { for all } r \text {, all } X \in \mathbf{G} .
$$

With the aid of $(4.2 \mathrm{a})$ and $(4.2 \mathrm{~b})$, we can write down the condition that $G^{\lambda}\left(H^{a, b}\right) \subset H^{a, b}$. Namely, we have: 
Theorem 4.2. $\mathrm{G}^{\lambda}$ leaves $H^{a, b}$ invariant if and only if:

$$
\lambda=\lambda_{b}=\lambda_{a-1} \text {. }
$$

If these conditions are satisfied, we have succeeded in analytically continuing the original representation $X \rightarrow X^{\prime}$ to a discrete set of reducible representations.

Conditions (4.2a) and (4.2b) can also be used to look for the Hermitian operator $A^{\lambda}$ described in Section 3 for which:

$$
X * A^{\lambda}+A^{\lambda} X=0 \text { for all } X \in \mathbf{G},
$$

i.e. can one find a new inner product $\left\{\psi / \psi^{\prime}\right\}=\left\langle\psi \mid A^{\lambda} \psi^{\prime}\right\rangle$ such that each of the operators $X^{\lambda}$ is skew-Hermitian with respect to the new inner product? Requiring that this new inner product be positive definite will give a restriction on the possible values of $\lambda$.

In fact, we will look for $A^{\lambda}$ of the simplest possible form:

$$
A^{\lambda}(\psi)=a_{r} \psi \text { for all } \psi \in H^{r}, r=0,1, \ldots
$$

By the definition of $\lambda_{r}$,

Hence,

$$
\begin{aligned}
& X^{\prime+} \psi=-\lambda_{r} X^{\prime \prime}+\psi, \\
& X^{\prime}-\psi=-\lambda_{r-1} X^{\prime \prime}-\psi .
\end{aligned}
$$

$$
\begin{aligned}
X^{\lambda} \psi= & X^{\prime \prime}+\left(\lambda-\lambda_{r}\right)+X^{\prime \prime}-\left(\lambda+\lambda_{r-1}\right)+X^{\prime 0}+\lambda X^{\prime \prime} 0(\psi) \\
X^{\lambda *} \psi= & X^{\prime \prime}+\left(\lambda+\lambda_{r}\right)+X^{\prime \prime}-\left(\lambda-\lambda_{r-1}\right)-X^{\prime 0}+\lambda X^{\prime \prime} 0(\psi) \\
X^{\lambda * A} \psi= & a_{r}\left(X^{\prime \prime}+\left(\lambda+\lambda_{r}\right)+X^{\prime \prime}-\left(\lambda-\lambda_{r-1}\right)+X^{\prime 0}+\lambda X^{\prime \prime}\right) \psi \\
A^{\lambda} X^{\lambda} \psi= & \left(a_{r+1} X^{\prime \prime}+\left(\lambda-\lambda_{r}\right)+a_{r-1} X^{\prime \prime}-\left(\lambda+\lambda_{r-1}\right)+\right. \\
& \left.+a_{r} X^{\prime 0}+a_{r} \lambda X^{\prime \prime}\right) \psi .
\end{aligned}
$$

Hence:

if and only if

$$
X^{\lambda *} A^{\lambda}+A^{\lambda} X^{\lambda}=0
$$

a) $a_{r}\left(\lambda+\lambda_{r}\right)=a_{r+1}\left(\lambda_{r}-\lambda\right), \quad r=0,1, \ldots$

b) $a_{r} X^{\prime \prime 0} H^{r}=0, \quad r=0,1, \ldots$

Condition b) is rather restrictive, since in general $X^{\prime \prime 0}$ will not be zero. However, this probably reflects the fact that supplementary seriestype representations do not always exist. One might ask: Why choose $A^{\lambda}$ of the simple form presented in (4.4)? One might note that this choice is forced if certain conditions are fulfilled. Suppose, for example, that $\mathbf{K}$ is a subalgebra of $\mathrm{G}$ such that:

a) $X \in \mathbf{K}$ is skew-Hermitian

b) $\mathbf{K} H^{r} \subset H^{r}$, and $\mathbf{K}$ acts irreducibly in $H^{r}$

c) Each irreducible representation of $\mathbf{K}$ occurs at most once in $H$.

Condition a) then forces:

$$
\left[A^{\lambda}, \mathbf{K}\right]=0 .
$$


Condition b) then forces:

$$
A^{\lambda} H^{r} \subset H^{r}
$$

while c) forces $A^{\lambda}$ to be of form (4.4).

\section{Remarks on the construction of multipliers}

Our method for constructing analytic continuations of representations has been dependent on assuming that a representation and a multiplier system for it is given. Now, in practice such multiplier systems are often given automatically when the representations are given "geometrically" by letting the group act on spaces of functions on homogeneous spaces of the group: The multipliers usually appear as the Jacobians of the transformations induced by the group elements. We will not get involved in this paper in geometric approach. However, there is a closely related algebraic formulation that we can investigate.

Suppose that $\mathbf{G}$ is a Lie algebra of operators on a Hilbert space $H$. However, we will not suppose that an $X \in G$ is a skew-Hermitian operator. (Note a slight variation from our previous notations: Here $X$ denotes both an element of the abstract Lie algebra $G$ and the operator on $H$ corresponding to it.) In fact, the deviation from skew-Hermiticity will provide us with the multipliers in favorable cases. In fact, let

$$
X^{\prime \prime}=\frac{1}{2}\left(X+X^{*}\right),
$$

i.e. $X^{\prime \prime}$ is the "Hermitian part" of $X$. Then, one might expect in certain cases that the assignment $X \rightarrow X^{\prime \prime}$ provides a multiplier system satisfying (2.3-4) relative to the originally given representation $X \rightarrow X$. In this case, $X \rightarrow X^{\prime}=X-X^{\prime \prime}$ is a skew-Hermitian representation for which $X \rightarrow X^{\prime \prime}$ again is a multiplier system, i.e. satisfies (2.3-4).

We will now provide a criterion that $X \rightarrow X^{\prime \prime}=\frac{1}{2}\left(X+X^{*}\right)$ provide a multiplier system in one important case. Suppose that $G$ can be written as $\mathbf{K} \oplus \mathbf{P}$, with :

$$
[\mathbf{K}, \mathbf{K}] \subset \mathbf{K} ;[\mathbf{K}, \mathbf{P}] \subset \mathbf{P} ;[\mathbf{P}, \mathbf{P}] \subset \mathbf{K} .
$$

Suppose also that:

$X$ is skew-Hermitian for $X \in \mathbf{K}$.

Then, for $X^{\prime}, Y \in \mathbf{P},[X, Y] \in \mathbf{K}$, hence:

But, also,

$$
[X, Y]^{*}=-[X, Y] \text {. }
$$

$$
\begin{aligned}
{[X, Y]^{*}-\left[X^{\prime}+X^{\prime \prime},\right.} & \left.Y^{\prime}+Y^{\prime \prime}\right]^{*} \\
& =\left(\left[X^{\prime}, Y^{\prime}\right]+\left[X^{\prime \prime}, Y^{\prime}\right]+\left[X^{\prime}, Y^{\prime \prime}\right]+\left[X^{\prime \prime}, Y^{\prime \prime}\right]\right)^{*} \\
& =\left[Y^{\prime}, X^{\prime}\right]-\left[Y^{\prime}, X^{\prime \prime}\right]-\left[Y^{\prime \prime}, X^{\prime}\right]+\left[Y^{\prime \prime}, X^{\prime \prime}\right] .
\end{aligned}
$$


Hence, it follows from (5.2) and (5.3) that:

$$
\left[X^{\prime \prime}, Y^{\prime}\right]+\left[X^{\prime}, Y^{\prime \prime}\right]=0 \quad \text { for } \quad X, Y \in \mathbf{P} .
$$

Also, for $X \in \mathbf{K}, Y \in \mathbf{P}$,

hence:

$$
[X, Y]^{*}=\left[Y^{*}, X^{*}\right]=-\left[Y^{*}, X\right]=\left[X, Y^{*}\right],
$$
a) $\left[X, Y^{\prime}\right]=[X, Y]^{\prime}$
b) $\left[X, Y^{\prime \prime}\right]=[X, Y]^{\prime \prime}$ for $X \in \mathbf{K}, Y \in \mathbf{P}$.

Then, we have:

Theorem 5.1. Suppose (5.2) and (5.3) are satisfied. Then, $X \rightarrow X^{\prime}$ $\left(=\frac{1}{2}\left(X-X^{\prime \prime}\right)\right)$ is a representation of $G$ if and only if

$$
\left[X^{\prime \prime}, Y^{\prime \prime}\right]=0 \text { for } X, Y \in \mathbf{P} \text {. }
$$

If this condition is satisfied, $X \rightarrow X^{\prime \prime}$ defines a multiplier system for the representation $X \rightarrow X^{\prime}$.

Suppose that (5.6) is satisfied. We would like to have further conditions that ( $4.2 \mathrm{a})$ be satisfied. Suppose that the Hilbert space $H$ is a direct sum

$$
H^{0}+H^{1}+H^{-1}+H^{2}+H^{-2}+\cdots
$$

of mutually orthogonal subspaces such that

b) $X H^{r} \subset H^{r-1}+H^{r}+H^{r+1}$ for $X \in \mathbf{P}$.

a) $\mathbf{K} H^{r} \subset H^{r}$ for all $r$.

For $X \in \mathbf{P}$, define $X^{\prime+}, X^{\prime 0}, X^{\prime-}, X^{\prime \prime}+, X^{\prime \prime} 0, X^{\prime \prime}$ - so that:

$$
\begin{aligned}
& X^{\prime+}, X^{\prime \prime}+\operatorname{map} H^{r} \text { into } H^{r+1} \\
& X^{\prime}-, X^{\prime \prime}-\text { are the adjoints of } X^{\prime+}, X^{\prime \prime}+ \\
& X^{\prime 0}, X^{\prime \prime 0} \text { maps } H^{r} \text { into } H^{r} . \\
& X^{\prime}+X^{\prime+}-X^{\prime}-+X^{\prime 0} \\
& X^{\prime \prime}=X^{\prime \prime}++X^{\prime \prime}-+X^{\prime \prime 0} .
\end{aligned}
$$

Now, $X^{\prime+}$ and $X^{\prime \prime}+$ both map $H^{r} \rightarrow H^{r+1}$, and, as $X$ varies over $\mathbf{P}$, both systems of maps have the same transformation properties under $K$. Assume further that:

The representation of $\mathrm{Ad} \mathbf{K}$ in $\mathbf{P}$ is irreducible.

In the tensor product $H^{r} \otimes H^{r+1 *}$, there is exactly one irreduc-

ible subspace that transforms under $\mathbf{K}$ like the representation

of $\operatorname{Ad} \mathbf{K}$ in $\mathbf{P}$. ( $H^{r+1 *}$ denotes the dual space to $H^{r+1}$.)

Obviously, a relation of the form $(4.2 \mathrm{a})$ follows from these two assumptions. 


\section{Construction of representations of $S L(2, R)$}

The group $S L(2, R)$ (or, $S U(1,1)$, which has the same Lie algebra) plays a key role in our work, since we are aiming to generalize to other groups results and methods that have been developed, usually in a very simple manner, for $S L(2, R)$. It is then interesting to show conversely how the general techniques apply to $S L(2, R)$. We will do this now.

Consider the unit circle in the complex $z$-phase, parameterized by the angle $\theta . S L(2, R)$ acts on this space. Let $H$ be the Hilbert space of square integrable periodic functions $\theta \rightarrow \psi(\theta)$, with the inner product:

$$
\left\langle\psi \mid \psi^{\prime}\right\rangle=\int_{0}^{2 \pi} \psi(\theta)^{*} \psi^{\prime}(\theta) d \theta
$$

Let $H^{r}, r=0, \pm 1, \pm 2, \ldots$ be the one-dimensional subspace spanned by $e^{i r \theta}$. Let $X$ be the operator on $H$ given by:

$$
Z=\frac{d}{d \theta}+i n, \quad n=\text { integer . }
$$

(The condition $n=$ integer is required in order that $\exp (t Z)$ map periodic functions into periodic functions.)

Let $X$ and $Y$ be the following operators:

$$
\begin{aligned}
& X=\sin \theta \frac{d}{d \theta}+i n \sin \theta . \\
& Y=\cos \theta \frac{d}{d \theta}+i n \cos \theta .
\end{aligned}
$$

Then,

$$
\begin{aligned}
{[Z, X] } & =Y ;[Z, Y]=-X . \\
{[X, Y] } & =\left[\sin \theta \frac{d}{d \theta}, \cos \theta \frac{d}{d \theta}\right] \\
& +i n\left(\left[\sin \theta, \cos \theta \frac{d}{d \theta}\right]+\left[\sin \theta \frac{d}{d \theta}, \cos \theta\right]\right) \\
& =-\left(\sin ^{2} \theta+\cos ^{2} \theta\right) \frac{d}{d \theta}+i n\left(-\cos ^{2} \theta-\sin ^{2} \theta\right) \\
& =-Z .
\end{aligned}
$$

We see that $X, Y$ and $Z$ generate the Lie algebra of $S L(2, R) . Z$ is skewHermitian, but $X$ and $Y$ are not:

$$
\begin{aligned}
& X^{*}=-\frac{d}{d \theta}(\sin \theta-i n \sin \theta=\cos \theta-X \\
& Y^{*}=\sin \theta-Y
\end{aligned}
$$

hence:

$$
\begin{aligned}
& X^{\prime \prime}=\frac{1}{2}\left(X+X^{*}\right)=-\frac{1}{2} \cos \theta . \\
& Y^{\prime \prime}=\frac{1}{2} \sin \theta .
\end{aligned}
$$


Note that $\left[X^{\prime \prime}, Y^{\prime \prime}\right]=0$, hence they form a multiplier system for the Lie algebra $(X, Y, Z)$.

Let us compute $\lambda_{r}$ as given by (4.5):

$$
\begin{aligned}
& X^{+}\left(e^{i r \theta}\right)=i r \cdot \frac{1}{2 i} e^{i \theta}+i n \cdot \frac{1}{2 i} e^{i \theta}\left(e^{i r \theta}\right) \\
&=\frac{1}{2}(r+n) e^{i \theta}\left(e^{i r \theta}\right) . \\
& X^{\prime \prime}+\left(e^{i r \theta}\right)=-\frac{1}{4} e^{i \theta}\left(e^{i r \theta}\right) \\
& Z^{-+}\left(e^{i r \theta}\right)=X^{+}-X^{\prime \prime}+\left(e^{i r \theta}\right)=\frac{(2 r+2 n+1)}{4} e^{i \theta}\left(e^{i r \theta}\right) \\
&=\frac{(2 r+2 n+1)}{4} \cdot-4 X^{\prime \prime}+\left(e^{i r \theta}\right), \quad \text { i.e. } \\
& \lambda_{r}=2 r+2 n+1,
\end{aligned}
$$

hence:

$$
\frac{a_{r}}{a_{r+1}}=\frac{2 r+2 n+1-\lambda}{\lambda+2 r+2 n+1} \text {. }
$$

Let us first check the conditions that $\frac{a_{r}}{a_{r+1}}>0$ for all $r$.

Then, either:

(a) $\lambda>2 r-2 n-1$ and $\lambda<2 r+2 n+1$

or

(b) $\lambda<-2 r-2 n-1$ and $\lambda>2 r+2 n+1$.

Case 1. $2 r+2 n+1 \geqq 0$.

Inequalities (b) are incompatible: (a) holding for all $r$ forces: $-1<\lambda<1$.

Case 2. $2 r+2 n-1<0$.

Similarly, $-1<\lambda<1$ is forced.

Thus, $\frac{a_{r}}{a_{r+1}}>0$ for all $r$ forces $-1<\lambda<1$, which corresponds to the supplementary series.

Now, let us look for the condition that $\frac{a_{r}}{a_{r+1}}>0$ for $r>0$. Notice that the subspace $H^{0}+H^{1}+H^{2}+\cdots$ of $H$ is that spanned by the boundary values on the unit circle of the functions that are complex analytic inside the unit circle, see that values of $\lambda$ for which $G^{\lambda}$ leaves this subspace invariant should correspond to the "discrete series". First then, we must have:

$$
X^{\lambda-H^{0}}=0
$$

Now,

$$
\begin{aligned}
X^{\lambda}-H^{0} & =\left(X^{\prime}-+\lambda X^{\prime \prime}\right) H^{0} \\
& =\left(-\lambda_{-1}+\lambda\right) X^{\prime \prime}-H^{0},
\end{aligned}
$$

or

$$
\lambda=\lambda_{-1}=2 n-1 \text {, }
$$


which is the condition that $\mathrm{G}^{\lambda}\left(H^{0}+H^{\prime}+\cdots\right) \subset H^{0}+H^{\prime}+\cdots$

$$
\frac{a_{r}}{a_{r+1}}=\frac{2 r+2 n+1-2 n+1}{2 n-1+2 r+2 n+1}=\frac{2 r+2}{4 r+2 n} \frac{r+1}{2 r+n} .
$$

Putting $r=0$ gives the condition:

$$
n \geqq 1 \text {. }
$$

Then, for each positive integer $n$ we obtain a skew-Hermitian representation $\mathrm{G}^{2 n-1}$ of the Lie algebra of $S L(2, R)$ in the space of functions that are complex analytic in the interior of the unit circle.

We can also look for the conditions that $\mathbf{G}^{\lambda}$ leaves invariant the subspace $H^{a}+H^{a+1}+\cdots+H^{b}$, with $a<b$. This would then give finite dimensional representations. Then, we must have

or

$$
\begin{aligned}
0=X^{\lambda-} H^{a} & =\left(X^{\prime}-+X^{\prime \prime}-\right) H^{a} \\
& =\left(\lambda-\lambda_{a-1}\right) X^{\prime \prime}-H^{a},
\end{aligned}
$$

$$
\text { Also, } \quad \begin{aligned}
0=X^{\lambda+} H^{b} & =\left(X^{\prime}++X^{\prime \prime}+\right)\left(H^{b}\right) \\
& =\left(\lambda-\lambda_{b}\right) X^{\prime \prime}+H^{b},
\end{aligned}
$$

or

$$
\lambda=\lambda_{b} \text {. }
$$

Using (6.1), $\quad 2 b+2 n+1=2(a-1)+2 n+1$, or

$$
a=b+1 \text {. }
$$

Finally, let us check the validity of the "Gell-Mann formula" $[3,4]$ for these representations. In this sample case, the requirement is that: $\left[\left[Z^{2}, X^{\prime \prime}\right],\left[Z^{2}, X^{\prime \prime}\right]\right]$ is a multiple of $Z$.

Now,

$$
\begin{aligned}
{\left[Z^{2}, X^{\prime \prime}\right] } & =Z\left[Z, X^{\prime \prime}\right]+\left[Z, X^{\prime \prime}\right] Z \\
& Z Y^{\prime \prime}+Y^{\prime \prime} Z \\
& =\left[Z, Y^{\prime \prime}\right]+2 Y^{\prime \prime} Z \\
& =-X^{\prime \prime}+2 Y^{\prime \prime} Z . \\
{\left[Z^{2}, Y^{\prime \prime}\right] } & =Z\left[Z, Y^{\prime \prime}\right]+\left[Z, Y^{\prime \prime}\right] Z \\
& =-Z X^{\prime \prime}-X^{\prime \prime} Z \\
& =-\left[Z, X^{\prime \prime}\right]-2 X^{\prime \prime} Z \\
& =-Y^{\prime \prime}-2 X^{\prime \prime} Z .
\end{aligned}
$$

$$
\begin{aligned}
{\left[\left[Z^{2}, X^{\prime \prime}\right],\left[Z^{2}, Y^{\prime \prime}\right]\right]=} & {\left[-X^{\prime \prime}+2 Y^{\prime \prime} Z,-Y^{\prime \prime}-2 X^{\prime \prime} Z\right] } \\
= & 2\left[X^{\prime \prime}, X^{\prime \prime} Z\right]-2\left[Y^{\prime \prime} Z, Y^{\prime \prime}\right]-4\left[Y^{\prime \prime} Z, X^{\prime \prime} Z\right] \\
= & -2 X^{\prime \prime} Y^{\prime \prime}+2 Y^{\prime \prime} X^{\prime \prime}-4\left(\left[Y^{\prime \prime} Z, X^{\prime \prime}\right] Z+\right. \\
& \left.+X^{\prime \prime}\left[Y^{\prime \prime} Z, Z\right]\right) \\
= & -4\left(Y^{\prime \prime 2} Z+X^{\prime \prime 2} Z\right) .
\end{aligned}
$$


Now, $Y^{\prime \prime 2}+X^{\prime \prime 2}$ is a Casimir operator of the Lie algebra generated by $Z, Y^{\prime \prime}$ and $X^{\prime \prime}$, hence is a scalar if the representation of this algebra is irreducible. We have then proved:

Theorem 6.1. Let $Z, X^{\prime \prime}, Y^{\prime \prime}$ be elements of a Lie algebra satisfying:

$$
\left[Z, X^{\prime \prime}\right]=Y^{\prime \prime} ;\left[Z, Y^{\prime \prime}\right]=-X^{\prime \prime} ;\left[X^{\prime \prime}, Y^{\prime \prime}\right]=0 \text {. }
$$

(The Lie algebra is then that of the group of rigid motions of the plane, which is a contraction of the Lie algebra of $S L(2, R))$. Consider them as operators on a Hilbert space with $Z$ skew-Hermitian, $X^{\prime \prime}$ and $Y^{\prime \prime}$ Hermitian, on which the Casimir operator $0=Y^{\prime \prime 2}+X^{\prime \prime 2}$ has the value $\delta^{2}$. Let

$$
X^{\prime}=\frac{1}{2 \delta}\left[Z^{2}, X^{\prime \prime}\right], Y^{\prime}=\frac{1}{2 \delta}\left[Z^{2}, Y^{\prime \prime}\right] .
$$

Then, $\left[X^{\prime}, Y^{\prime}\right]=-Z$, i.e., $\left(Z, X^{\prime}, Y^{\prime}\right)$ generates the Lie algebra of $S L(2, R)$ : The Gell-Mann formula holds, and enables one to pass from a representation of the contracted non-semi-sinple group back to a representation of the semi-simple group.

There is another more abstract approach to this result that is independent of representations. Consider the Lie algebra $G^{\prime \prime}$ generated by elements $X^{\prime \prime}, Y^{\prime \prime}, Z$, with

$$
\left[Z, X^{\prime \prime}\right]=Y^{\prime \prime} ;\left[Z, Y^{\prime \prime}\right]=-X ;\left[X^{\prime \prime}, Y^{\prime \prime}\right]=0 \text {. }
$$

As mentioned in [4] in connection with the group-theoretic treatment of the hydrogen atom, one can construct the "complete" universal enveloping algebra consisting of all "functions" of the generators of $\mathbf{G}^{\prime}$. (The universal enveloping algebra used in Lie algebra theory just involves polynomials in the generators. The algebraic foundations of this construction will be dealt with in a later paper.) In terms of this algebra one can construct the generators of the Lie algebra of $S L(2, R)$ : Define

$$
\begin{aligned}
& X=\left[Z^{2}, X^{\prime \prime}\right] / 2\left(X^{\prime \prime 2}+Y^{\prime \prime 2}\right)^{1 / 2} \\
& Y=\left[Z^{2}, X^{\prime \prime}\right] / 2\left(X^{\prime \prime 2}+Y^{\prime \prime 2}\right)^{1 / 2}
\end{aligned}
$$

As above, one proves that:

$$
[X, Y]=-Z ;[Z, X]=Y ;[Z, X]=-Y,
$$

i.e. $(Z, X, Y)$ generate the Lie algebra of $S \mathbf{G}(2, R)$. One might conjecture that there is a similar relation between any Lie algebra $\mathbf{G}$ and one of its contractions $\mathbf{G}^{\prime}$, i.e. one can define $\mathbf{G}$ as a subalgebra of this complete universal enveloping algebra of $\mathbf{G}^{\prime}$.

\section{Another method for constructing hermitian multipliers}

Suppose now that $\mathbf{G}$ is a Lie algebra, admitting a Cartan-type decomposition:

$$
\mathbf{G}=\mathbf{K} \oplus \mathbf{P} ;[\mathbf{K}, \mathbf{K}] \subset \mathbf{K} ;[\mathbf{K}, \mathbf{P}] \subset \mathbf{P} ;[\mathbf{P}, \mathbf{P}] \subset \mathbf{K} .
$$


Suppose that $G$ admits a representation $X \rightarrow X^{\prime}$ by skew-Hermitian operators on a Hilbert space $H$. Assume that $H$ admits a decomposition

$$
H^{0}+H^{1}+H^{-1}+H^{2}+H^{-2}+\cdots,
$$

and that $X^{\prime}$, for $X \in \mathbf{P}$, can be decomposed as usual onto the form:

and that $\mathbf{K} H^{r} \subset H^{r}$.

$$
X^{\prime+}-X^{\prime}-+X^{\prime 0}
$$

For $X, Y \in \mathbf{P}$,

$$
\begin{aligned}
{[X, Y]=} & {\left[X^{\prime+}-X^{\prime}-+X^{\prime 0}, Y^{\prime+}-Y^{1-}+Y^{\prime 0}\right] } \\
= & {\left[X^{\prime+}, Y^{\prime+}\right]+\left[X^{\prime}-, Y^{\prime}-\right] } \\
& -\left[X^{\prime+}, Y^{\prime}\right]-\left[X^{\prime}-, Y^{\prime+}\right]+\left[X^{\prime 0}, Y^{\prime 0}\right] \\
& +\left[X^{\prime 0}, Y^{\prime+}\right]+\left[X^{\prime+}, Y^{\prime} 0\right] \\
& -\left[X^{\prime}-, Y^{\prime} 0\right]-\left[X^{\prime 0}, Y^{\prime}-\right] .
\end{aligned}
$$

This gives the following relations:

a) $[X, Y]=-\left[X^{\prime+}, Y^{\prime-}\right]-\left[X^{\prime}-, Y^{\prime+}\right]+\left[X^{\prime 0}, Y^{\prime 0}\right]$.

b) $0=\left[X^{\prime+}, Y^{\prime+}\right]=\left[X^{\prime}-Y^{\prime-}\right]$

c) $\left[X^{\prime 0}, Y^{\prime+}\right]+\left[X^{\prime+}, Y^{\prime 0}\right]=0$

d) $\left[X^{\prime-}, Y^{\prime 0}\right]+\left[X^{\prime 0}, Y^{\prime}-\right]=0$.

Let $\alpha_{r}, r=0, \pm 1, \pm 2, \ldots$ be a sequence of real numbers.

For $X \in \mathbf{P}$, define $X^{\prime \prime}=X^{\prime \prime}++X^{\prime \prime}-$ as follows:

$$
\begin{aligned}
& \text { a) } X^{\prime \prime}+\psi=\alpha_{r} X^{\prime+} \psi \text { for } \psi \in H^{r} . \\
& \text { b) } X^{\prime \prime}-\psi=\alpha_{r-1} X^{\prime}-\psi \text { for } \psi \in H^{r} .
\end{aligned}
$$

It is readily seen that $X^{\prime \prime}$ - is the adjoint of $X^{\prime \prime+}$, so that $X^{\prime \prime}$ is Hermitian. Further, for $Y \in \mathbf{K}$,

hence

$$
\begin{aligned}
& {\left[Y, X^{\prime \prime+}\right]=[Y, X]^{\prime \prime+},} \\
& {\left[Y, X^{\prime \prime}-\right]=[Y, X]^{\prime \prime-},}
\end{aligned}
$$

$$
\left[Y, X^{\prime \prime}\right]=[Y, X]^{\prime \prime} \text {. }
$$

Now, for $X, Y \in \mathbf{P}$, let us compute:

$$
\left[X^{\prime \prime}, Y^{\prime \prime}\right]=\left[X^{\prime \prime+}, Y^{\prime \prime+}\right]+\left[X^{\prime \prime}-, Y^{\prime \prime-}\right]+\left[X^{\prime \prime+}, Y^{\prime \prime}-\right]+\left[X^{\prime \prime}-, Y^{\prime \prime+}\right] \text {. }
$$

The first two terms are zero, from 7.1 a) and b)

or

$$
\begin{aligned}
& X^{\prime \prime}+Y^{\prime \prime}-H^{r}=X^{\prime \prime}+\alpha_{r-1} Y^{\prime}-H^{r}=\alpha_{r-1}^{2} X^{\prime}+Y^{\prime}-H^{r} \\
& Y^{\prime \prime}-X^{\prime \prime}+H^{r}=Y^{\prime \prime}-\alpha_{r} X^{\prime}+H^{r}=\alpha_{r}^{2} Y^{\prime}-X^{\prime}+H^{r}
\end{aligned}
$$

$$
\begin{aligned}
{\left[X^{\prime \prime}+, Y^{\prime \prime}-\right] H^{r} } & =\left(\alpha_{r-1}^{2} X^{\prime}+Y^{\prime}--\alpha_{r}^{2} Y^{\prime}-X^{\prime+}\right) H^{r} . \\
{\left[X^{\prime \prime}-, Y^{\prime \prime+}\right] H^{r} } & =-\left[Y^{\prime \prime}+, X^{\prime \prime}-\right] \\
& \equiv\left(\alpha_{r-1}^{2} Y^{\prime}+X^{\prime}--\alpha_{r}^{2} X^{\prime}-Y^{\prime+}\right) H^{r} .
\end{aligned}
$$


Thus, in order that $\left[X^{\prime \prime}, Y^{\prime \prime}\right]=0$, we must have:

$$
\alpha_{r-1}^{2}\left(X^{\prime}+Y^{\prime}--Y^{\prime}+X^{\prime-}\right)-\alpha_{r}^{2}\left(Y^{\prime}-X^{\prime}+-X^{\prime-} Y^{\prime+}\right) H^{r}=0 .
$$

Now,

$X^{\prime}+Y^{\prime}--Y^{\prime}+X^{\prime-}=\left[X^{\prime}+, Y^{\prime-}\right]-\left[Y^{\prime+}, X^{\prime-}\right]+Y^{\prime}-X^{\prime}+-X^{\prime}-Y^{\prime-}$,

hence, using $7.2 \mathrm{a})$,

$$
\begin{aligned}
\left(\alpha_{r-1}^{2}-\alpha_{r}^{2}\right)\left(Y^{\prime}-X^{\prime}+\right. & \left.-X^{\prime}-Y^{\prime+}\right)+ \\
& +\alpha_{r-1}^{2}\left(-\left[X, Y^{\prime}\right]+\left[X^{\prime 0}, Y^{\prime 0}\right]\right) H^{r}=0 .
\end{aligned}
$$

Notice the meaning of this condition: Assigning to $X, Y \in \mathbf{P}$, the operators $\left[X^{\prime 0}, Y^{\prime 0}\right]-[X, Y]$ and $Y^{\prime}-X^{\prime}+-X^{\prime}-Y^{\prime}+$ defines two bilinear mappings of $\mathbf{P} \times \mathbf{P}$ to the skew-Hermitian operators on $H$ ? Further, these systems transform in the same way under the action of $K$. (7.4) then requires a linear relation between them, which involves a generalization of what the physicists call the "Wigner-Eckart Theorem".

This gives one of the necessary conditions that $X \rightarrow X^{\prime \prime}$ define a multiplier system. The other is:

Now,

$$
\left[X^{\prime \prime}, Y^{\prime}\right]+\left[X^{\prime}, Y^{\prime \prime}\right]=0 \text {. }
$$

$$
\begin{aligned}
{\left[X^{\prime \prime}, Y^{\prime}\right] } & =\left[X^{\prime \prime}++X^{\prime \prime}-, Y^{\prime+}-Y^{\prime-}\right] \\
& =\left[X^{\prime \prime}+, Y^{\prime+}\right]-\left[X^{\prime \prime}-, Y^{\prime-}\right]+\left[X^{\prime \prime}-, Y^{\prime+}\right]-\left[X^{\prime \prime}+, Y^{\prime-}\right] .
\end{aligned}
$$

Hence,

$$
\begin{aligned}
{\left[X^{\prime \prime}, Y^{\prime}\right]+\left[X^{\prime}, Y^{\prime \prime}\right]=} & {\left[X^{\prime \prime}, Y^{\prime}\right]-\left[Y^{\prime \prime}, X^{\prime}\right] } \\
= & {\left[X^{\prime \prime}+, Y^{\prime+}\right]-\left[X^{\prime \prime}-, Y^{\prime}-\right]+\left[X^{\prime \prime}-, Y^{\prime+}\right]-} \\
& -\left[X^{\prime \prime}+, Y^{\prime}-\right]-\left[Y^{\prime \prime}+, X^{\prime+}\right]+\left[Y^{\prime \prime}-, X^{\prime}-\right]- \\
& -\left[Y^{\prime \prime}-, X^{\prime+}\right]+\left[Y^{\prime \prime}+, X^{\prime}-\right] .
\end{aligned}
$$

Hence, (7.5) requires:

a) $\left[X^{\prime \prime}-, Y^{\prime-}\right]=\left[Y^{\prime \prime}-, X^{\prime-}\right]$

b) $\left[Y^{\prime \prime}+, X^{\prime+}\right]=\left[X^{\prime \prime}+, Y^{\prime+}\right]$

c) $\left[X^{\prime \prime}-, Y^{\prime+}\right]-\left[X^{\prime \prime}+, Y^{\prime-}\right]=\left[Y^{\prime \prime}-, X^{\prime+}\right]-\left[Y^{\prime \prime}+, X^{\prime-}\right]$.

We will now check these identities:

$$
\begin{aligned}
& X^{\prime \prime}+Y^{\prime}+H^{r}=\alpha_{r+1} X^{\prime}+Y^{\prime}+H^{r} \\
& Y^{\prime}+X^{\prime \prime}+H^{r}=\alpha_{r} Y^{\prime}+X^{\prime}+H^{r} \\
& Y^{\prime \prime}+X^{\prime}+H^{r}=\alpha_{r+1} Y^{\prime}+X^{\prime}+H^{r} \\
& X^{\prime}+Y^{\prime \prime}+H^{r}=\alpha_{r} X^{\prime}+Y^{\prime}+H^{r}
\end{aligned}
$$

(7.6b) then follows from (7.2b). (7.6a) follows from b) after taking the adjoint. 
Now, let us proceed to $\mathrm{c})$.

hence :

$$
\begin{aligned}
X^{\prime \prime}-Y^{\prime}+H^{r}= & \alpha_{r} X^{\prime}-Y^{\prime}+H^{r} \\
Y^{\prime}+X^{\prime \prime}-H^{r}= & \alpha_{r-1} Y^{\prime}+X^{\prime}-H^{r} . \\
\left(\left[X^{\prime \prime}-, Y^{\prime+}\right]-\left[Y^{\prime \prime}-, X^{\prime}+\right]\right) H^{r}= & \alpha_{r}\left(X^{\prime}-Y^{\prime}+-Y^{\prime}-X^{\prime}+\right) H^{r}- \\
& -\alpha_{r-1}\left(Y^{\prime}+X^{\prime}--X^{\prime}+Y^{\prime}-\right) H^{r} . \\
X^{\prime \prime}+Y^{\prime}-H^{r}= & \alpha_{r-1} X^{\prime}+Y^{\prime}-H^{r} . \\
Y^{\prime}-X^{\prime}+H^{r}= & \alpha_{r} Y^{\prime}-X^{\prime}+H^{r}
\end{aligned}
$$

$$
\begin{aligned}
\left(\left[X^{\prime \prime}+, Y^{\prime-}\right]-\left[Y^{\prime \prime}+, X^{\prime-}\right]\right) H^{r}= & \alpha_{r-1}\left(X^{\prime}+Y^{\prime}-Y^{\prime+} X^{\prime-}\right) H^{r} \\
& +\alpha_{r}\left(Y^{\prime}-X^{\prime}+-X^{\prime}-Y^{\prime+}\right) H^{r} .
\end{aligned}
$$

We see then that $(7.6 \mathrm{c})$, hence also (7.5), is automatically satisfied, with no special conditions on $\alpha_{r}$. (7.4) then is the only restrictive assumption on $\alpha_{r}$. We will leave these calculations at this point: The qualitative point we have made can be described as follows:

Theorem 7.1. Suppose that in the linear space of bilinear mappings $\mathbf{P} \times \mathbf{P} \rightarrow$ (skew Hermitian operators on $H^{r}$ ) there is just one subspace transforming under $K$ as the one described by: $(X, Y) \rightarrow\left[X^{\prime 0}, Y^{\prime 0}\right]-$ $-\left[X^{\prime}, Y^{\prime}\right]$. Then, there is as Hermitian multiplier system for the representation, which connects $H^{r}$ with $H^{r-1}$ and $H^{r+1}$, and hence the representation can be analytically continued. Further, two such multiplier systems which are normalized to be equal on $H^{0}$ can only differ by a change of sign on each of the other subspaces $H^{r}$.

\section{Remarks on the relations between finite and infinite dimensional representations of non-compact semi-simple groups}

Let $G$ be a non-compact semi-simple Lie group with finite center, and let $K$ be its maximal compact subgroup. There is a general theorem in representation theory [6] asserting that the number of times a given irreducible representation of $K$ appears in an irreducible unitary representation of $G$ is majorized by the maximal number of times this representation of $K$ may appear in the finite dimensional irreducible representations of $G$. This result no doubt has a close relation to analytic continuation: Certainly, it is obvious without further proof that the simple types of analytic continuation considered in this paper satisfy this condition, since the whole Hilbert space is built up from finite dimensional representations.

It is also possible and useful to use analytical continuation to obtain information about the finite dimensional representation in terms of facts that are known about the infinite dimensional one. We will look at one such typical situation. 
Suppose that we ask: How does one construct finite dimensional representations of $G$ that have the property that each irreducible representation of $K$ appears at most once when the representation of $G$ is reduced with respect to $K$ ? For example, such a question plays an important foundational role in the choice of $S U(3)$ to classify elementary particles: For the eight spin 1/2 baryons for example, the exact symmetry of the Hamiltonian is $S U(2) \times U(1)$ ("isospin" and "hypercharge"). One reason that the group $S U(3)$ containing them is a good choice for use in physics in constructing multiplies is the fact that in each irreducible representation of $S U(3)$ the representations of $S U(2) \times$ $\times U(1)$ appear at most once. B. Kostant has proved (unpublished) that the only pairs $(G, K)$ of a compact group $G$ and a subgroup $K$ with this property are the choices:

$$
\begin{aligned}
& G=S U(n), K=S U(n-1) \times U(1) \\
& G=S O(n), \quad K=S O(n-1) .
\end{aligned}
$$

Now, it is quite easy to quote many examples of unitary representations of $G$ which have this property (see [3]): A theorem proved by E. CARTAN suggests looking for them by examining the symmetric spaces $K / L$ on which the action of $K$ can be extended to give an action of $G$. Similarly, one may hope to link together the finite and infinite dimensional representations of $G$ in which $K$ has a one-dimensional invariant subspace (which could be taken as $H^{0}$ ) by analytic continuation.

\section{Relations between $S L(2, R)$ and the Heisenberg group}

In Section 6 , we pointed out the relations between $G$, the Lie algebra of $S L(2, R)$, and one of its contractions, the Lie algebra of the group of rigid motions of the plane. In this section we will consider another of its contractions, the Heisenberg algebra, and discuss the corresponding limits of representations.

Let $\mathbf{G}$ be the three-dimensional Lie algebra generated by $X, Y, Z$, and commutation relations:

$$
\begin{aligned}
& {[X, Y]=-Z} \\
& {[Z, X]=Y,[Z, Y]=-X .}
\end{aligned}
$$

The Heisenberg algebra $\mathbf{G}^{\prime}$ is isomorphic as a vector space to $\mathbf{G}$, i.e., is generated by three elements $X^{\prime}, Y^{\prime}, Z^{\prime}$, with the commutation relations:

$$
\begin{aligned}
{\left[Z^{\prime}, X^{\prime}\right] } & =0=\left[Z^{\prime}, Y\right] \\
{\left[X^{\prime}, Y^{\prime}\right] } & =-Z .
\end{aligned}
$$

(Usually, of course, $Z^{\prime}$ is labelled $i, Y$ labelled $p, X^{\prime}$ labelled $q$, so that $[p, q]=i$, which is the familiar Heisenberg relation.) This algebra can be obtained by the contraction process [3] from $\mathbf{G}$. 
For $\varepsilon>0$, define $\alpha_{\varepsilon}: \mathbf{G} \rightarrow \mathbf{G}$ as follows:

$$
\begin{aligned}
\alpha_{\varepsilon}(Z) & =\varepsilon Z \\
\alpha_{\varepsilon}(X) & =\sqrt{\varepsilon} X ; \alpha_{\varepsilon}(Y)=\sqrt{\varepsilon} Y .
\end{aligned}
$$

Let $[,]^{\prime}$ be the Lie algebra structure resulting from the contraction process relative to the one-parameter family $\varepsilon \rightarrow \alpha_{\varepsilon}$. As definition:

Similarly, $[Z, Y]^{\prime}=0$.

$$
\begin{aligned}
{[Z, X]^{\prime} } & =\lim _{\varepsilon \rightarrow 0} \alpha_{\varepsilon}^{-1}\left[\alpha_{\varepsilon}(Z), \alpha_{\varepsilon}(X)\right] \\
& =\lim _{\varepsilon \rightarrow 0} \sqrt{\varepsilon^{-1}}[\varepsilon Z, \sqrt{\varepsilon} X]=0 .
\end{aligned}
$$

$$
\begin{aligned}
{[X, Y]^{\prime} } & =\lim _{\varepsilon \rightarrow 0} \alpha_{\varepsilon}^{-1}\left[\alpha_{\varepsilon}(X), \alpha_{\varepsilon}(Y)\right] \\
& =-Z .
\end{aligned}
$$

We see that this contracted Lie algebra is isomorphic to the Heisenberg algebra.

Now we can see that the discrete series of representations of $S L(2, R)$ described in Section 6 goes over in the limit as $n \rightarrow \infty$ to a representation of the Heisenberg algebra. Recall that:

$$
\begin{aligned}
& Z_{n}=\frac{d}{d \theta}+i n . \\
& X_{n}=\sin \theta \frac{d}{d \theta}+i n \sin \theta-\frac{(2 n-1)}{2} \cos \theta . \\
& Y_{n}=\cos \theta \frac{d}{d \theta}+i n \cos \theta+\frac{(2 n-1)}{2} \sin \theta .
\end{aligned}
$$

At first sight it looks like the limiting operators are trivial, since $\frac{Z_{n}}{n}, X_{n} / n, Y_{n} / n$ all go over, as $n \rightarrow \infty$, to abelian operators. However there is a new feature here: The inner product on the Hilbert space is also changing with $n$. The Hilbert space itself must be changed by a linear transformation in order to compare the limit as $n \rightarrow \infty$.

Recall that the Hilbert space is the sum $H^{0}+H^{\prime}+\cdots$. The inner product $\left\langle\psi \mid \psi^{\prime}\right\rangle_{n}$ at the $n$-th stage is

with

$$
a_{r}\left\langle\psi / \psi^{\prime}\right\rangle, \text { for } \psi, \psi^{\prime} \in H^{r},
$$

$$
\frac{a_{r+1}}{a_{r}}=\frac{2 r+n}{r+1}
$$

Let $A_{n}$ be the Hermitian operator that sends $\psi \in H^{r}$ into $a_{r} \psi$. The $n$-th stage inner product is then

$$
\left\langle\psi \mid A_{n} \psi^{\prime}\right\rangle=\left\langle\psi / \psi^{\prime}\right\rangle_{n}
$$

Let $B_{n}$ be a linear map of the Hilbert space into itself that effects an isomorphism between the original Hilbert space and the $n$-th one.

$$
\left\langle B_{n} \psi \mid B_{n} \psi\right\rangle=\left\langle\psi \mid A_{n} \psi\right\rangle \text {. }
$$


Thus, $B_{n}$ can be taken as:

Then the operators

$$
B_{n} \psi=\sqrt{a_{r}} \psi \text { for } \psi \in H^{r} .
$$

$$
B_{n} X_{n} B_{n}^{-1}, B_{n} Y_{n} B_{n}^{-1}, B_{n} Z_{n} B_{n}^{-1}
$$

are skew-Hermitian with respect to the original Hilbert space structure $\left\langle\psi \mid \psi^{\prime}\right\rangle$, and satisfy the commutation relations (8.1).

Notice first that:

hence

$$
B_{n} Z_{n} B_{n}^{-1}=Z_{n}
$$

$$
\begin{aligned}
& \quad \frac{B_{n} Z B_{n}^{-1}}{n} \rightarrow i \quad \text { as } n \rightarrow \infty \\
& B_{n} X_{n} B_{n}^{-1}\left(e^{i r \theta}\right)=\alpha_{r}^{-1 / 2} B_{n} X_{n}\left(e^{i r \theta}\right) \\
&=\alpha_{r}^{-1 / 2} B_{n}\left[\sin \theta\left(i r e^{i r \theta}+i n e^{i r \theta}\right)-\frac{2 n-1}{2} \cos \theta e^{i r \theta}\right] \\
&=\alpha_{r}^{-1 / 2} B_{n}\left[\frac{1}{2}\left(e^{i(r+1) \theta}-e^{i(r-1) \theta}(r+n)-\frac{2 n-1}{4}\left(e^{i(r+1) \theta}+e^{i(r-1) \theta}\right)\right]\right. \\
&=\alpha_{r}^{-1 / 2}\left[\alpha_{r+1}^{1 / 2} e^{i(r+1) \theta}\left(\frac{r+n}{2}-\frac{2 n-1}{4}\right)-\right. \\
&-\alpha_{r}^{1 / 2} e^{i(r-1) \theta}\left(\frac{r+n}{2}+\frac{2 n-1}{4}\right) \\
&=\left(\frac{\alpha_{r+1}}{\alpha_{r}}\right)^{1 / 2} e^{i(r+1) \theta} \frac{2 r+1}{4}-\left(\frac{\alpha_{r-1}}{\alpha_{r}}\right)^{1 / 2} e^{i(r-1) \theta} \frac{2 r+4 n-1}{4} \\
&=\left(\frac{2 r+n}{r+1}\right)^{1 / 2} e^{i(r+1) \theta} \frac{2 r+1}{4}-\left(\frac{r}{2 r-2+n}\right)^{1 / 2} e^{i(r-1) \theta} \frac{2 r+4 n-1}{4} .
\end{aligned}
$$

Then,

$$
\frac{B_{n} X_{n} B_{n}^{-1}\left(e^{i r \theta}\right)}{n^{1 / 2}} \rightarrow \frac{(2 r+1)}{4} e^{i(r+1)} \theta-r^{1 / 2} e^{i(r-1) \theta} \quad \text { as } \quad n \rightarrow \infty .
$$

This one-parameter family of representations of $S L(2, R)$ then contracts, as predicted by (9.2), into a representation of the Heisenberg group, whereas the principal series of representations of $S L(2, R)$ contracts into a representation of the other contracted algebra, that of the group of rigid motions in the plane. One also obtains in this way asymptotic relations for the matrix elements of the corresponding representations of the group. Obviously this procedure can be applied to much more general groups than $S L(2, R)$. This will be done in more detail in a later paper.

\section{The $S U(6)$ quark model as a representation of $S U(3,2)$}

One of the minor mysteries of the $S U(6)$-theory of GURSEY, RADACATI and SAKITA is the fact that the baryons fit into the 56 dimensional representation of $S U(6)$, i.e. the symmetric tensor representation, instead of in the 20 , i.e. the skew-symmetric tensors, as one would expect of fermions. This is analogous to the also-unexplained question why the 
physically relevant representations of $S U(3)$ are those in which the center $Z_{3}$ is represented as the identity.

One might ask whether there is some relation of this to a grouptheoretical property that might provide a clue to the physical explanation. Now, there is one such obvious explanation for the $S U(3)$-property: $S U(3)$ is the maximal compact subgroup of $S L(3, C)$, and the most obvious family of irreducible unitary representations of $S L(3, C)$ (the "principal degenerate series") has the property that the representations of $S U(3)$ appearing in its decomposition form a "ladder" for which the 8,10 and 27 dimensional representations of $S U(3)$ form the first few "rungs", with each representation of $S U(3) / Z_{3}$ appearing exactly once. In turn, this is connected with the geometric action of $S U(3)$ modulo its center on $P^{2}(C)$, two dimensional complex projective space.

The $S U(6)$ theory seems more complicated from a group-theoretical point of view. I know of no non-compact group containing $S U(6)$ that seems suitable for explaining in a strictly analogous way the pattern of representations of $S U(6)$ that seem physically useful. (Except of course for $S U(6,6)$, but this has a different flavor since it incorporates the Lorentz group, and seems therby to lead to severe difficulties in physical interpretation.) We will now show that consideration of $S U(3,2)$ leads in a very natural way to many of the attractive features of classification of particles by $S U(6)$, although at first sight it has nothing to do with $S U(6)$.

I would like to thank S. GLASHOw and E. StEIN, who suggested studying $S U(3,2)$.

Let $G=S U(3,2)$, i.e. the group of $5 \times 5$ complex matrices leaving invariant a Hermitian form with 3 plus and 2 minus signs. Then $K$, the maximal compact subgroup of $G$, is $S U(3) \times S U(2) \times U(1)$. For purposes of classifying elementary particles, we intend to identify the $S U(3)$ subgroup with unitary spin, $S U(2)$ with the spin associated with the Lorentz group, and $U(1)$ with baryon number. We will consider an irreducible representation of $\mathbf{G}$ by skew-Hermitian operators on a Hilbert space $H$ such the $\mathbf{K}$ has an invariant vector $\psi_{0}$. Let $\mathbf{G}=\mathbf{K} \oplus \mathbf{P}$ be the Cartan decomposition. Let $\mathbf{P}_{c}=\mathbf{P}+i \mathbf{P}$ be the complexification of $\mathbf{P}$. Following a suggestion by E. ThIELEKer [5] $H$ can be built up by applying operators of $\mathbf{P}_{c}$ to $\psi_{0}$. Consider such a product:

$$
X_{1} \ldots X_{r} \psi_{0}, \text { with } X_{1}, \ldots, X_{r} \in \mathbf{P}_{c} .
$$

Since $\left[\mathbf{P}_{c}, \mathbf{K}_{c}\right] \subset \mathbf{K}_{c},\left[\mathbf{P}_{c}, \mathbf{P}_{c}\right] \subset \mathbf{K}_{c}$, one sees that only the symmetric product of the $X$ 's matters, i.e. $H$ reduces under $\mathbf{K}$ into subspaces which are obtained by reducing the symmetric tensor products of the adjoint representation of $K$ in $\mathbf{P}_{c}$. 
For $G=S U(3,2)$, it is readily seen that $\operatorname{Ad} K$ acting in $\mathbf{P}_{c}$ is not irreducible (since $K$ has a non-discrete center, namely $U(1)$ ). In fact, $\mathbf{P}_{c}$ splits up into the direct sum of subspaces $\mathbf{P}^{+} \oplus \mathbf{P}^{-}$, in each of which $\operatorname{Ad} K$ acts irreducibly, with $\mathbf{P}^{-}$the complex conjugate of $\mathbf{P}^{+}$, and such that $U(1)$ acts in $\mathbf{P}^{+}$by multiplication by $\exp (i \theta / 3)$, in $\mathbf{P}^{-}$by $\exp (-i \theta / 3)$. (The factor $1 / 3$ is just a normalization, suggested by the physics, since $U(1)$ is to represent baryon number.)

Let us denote a typical element of $\mathbf{P}^{+}$by $X$, a typical element of $\mathbf{P}-$ by $\bar{X}$. We can consider $\psi_{0}$ as the "vacuum state", the vectors $X \psi_{0}$ as the "quarks", the vectors $\bar{X} \psi_{0}$ as the "antiquarks", to which we assign baryon number $1 / 3$ and $-1 / 3$. It is readily seen that the representation of $S U(3) \times S U(2)$ on $\mathbf{P}^{+}$is the tensor product of the 3 and 2 dimensional representation of $S U(3)$ and $S U(2)$, respectively, which we denote by: $3 \times 2$. Then, the representation in $\mathbf{P}^{-}$is $\overline{3} \times 2$ (since $\overline{2}=2$ ). Now, $\operatorname{dim} \mathbf{P}^{+}=6$, hence it can be considered as the space of the basic representation of $S U(6)$, with $S U(3) \times S U(2)$ appearing as a subgroup of $S U(6)$ in a manner identical to that in the Gursey-Radicati-Sakita theory.

According to the general theory of [5] all of $H$ can be built up as symmetric products of the quarks and antiquarks, exactly as the elementary particles are built up in the $S U(6)$ quark model. For example, consider vectors of the form

$$
\bar{X} X \psi_{0} \text {. }
$$

They form the 35 dimensional representation of $S U(6)$, which is identified with the mesons. Note that baryon number and spin is correct under this assignment: $U(1)$ leaves these vectors invariant, and the center of $S U(2)$ acts as the identity, i.e. these vectors have integral spin.

The vectors of the form

$$
X X X \psi_{0}
$$

form the 56 dimensional representation of $S U(6)$, which is identified with the baryons. Note again the assignment of baryon number and spin are correct: $U(1)$ acts by multiplication by $\exp (i \theta)$, which we identify with baryon number 1 , and the center of $S U(2)$ acts as minus the identity, i.e. the $S U(2)$-content is that of half-integer spin.

The new feature that is possibly attractive from the physical point of views is that the operators of $\mathbf{P}$ tie together the various multiples built up out of quarks. Further, $S U(2) \times S U(3) \times U(1)$ is a symmetric subgroup of $S U(3,2)$, hence from a group theoretic point of view the construction is closer to that by which one passes from $S U(2) \times U(1)$ (isospin $\times$ hypercharge) to $S U(3)$ than the standard way of considering $S U(6)$ directly.

Finally, we may mention that the geometric method may be used to give essentially the same results [4], [6]. $G$ can be made to act on $\mathbf{P}^{+}$ 
via linear fractional transformations; hence $G$ acts on the polynomial functions of $\mathbf{P}^{+}$. Complex coordinates $\left(z_{1}, \ldots, z_{6}\right)$ can be introduced for $\mathbf{P}^{+}$so that $K$ acts via unitary matrices. Polynomials of $\mathbf{P}^{+}$are then combinations of products of the $z^{\prime} s$ and $\bar{z}^{\prime} s$.

\section{Bibliography}

[1] Bargmann, V.: Irreducible representations of the Lorentz group. Ann. Math. 48,568 (1947).

[2] Gel'Fand, I. M., and M. A. NedmaRK: Unitare Darstellungen der Klassischen Gruppen. Berlin: Akademie-Verlag 1957.

[3] Hermann, R.: Lie groups for physicists. New York: W. A. Benjamin, Inc. 1965.

[4] - Some properties of representatives of non-compact groups. To appear. Proceedings of the Seminar on Elementary Particles, International Center for Theoretical Physics, Trieste, 1965.

[5] - Group representations and creation operators. Commun. Math. Phys. 2, $18-93$ (1966).

[6] Stein, E.: Lectures on group representations. To appear. Proceedings of the Seminar on Elementary Particles, International Center for Theoretical Physics, Trieste, 1965. 